\title{
Determinants of Acute Diarrhoea among Children Aged 6-59 Months in Chire District, Southern Ethiopia: Unmatched Case-Control Study
}

\author{
Gorfu Geremew Gunsa ${ }^{1}$, Kaleb Mayisso Rodamo ${ }^{2,}$, , Desalegn Dabaro Dangiso ${ }^{3}$ \\ ${ }^{1}$ Chire Hospital, Hawassa, Ethiopia \\ ${ }^{2}$ Faculty of Health Sciences, College of Medicine and Health Sciences, Hawassa University, Hawassa, Ethiopia \\ ${ }^{3}$ Department of Social and Population Health, Yirgalem Hospital Medical College, Yirgalem, Ethiopia \\ Email address: \\ gorfugeremew@gmail.com(G. G. Gunsa),kalebmayisso@gmail.com(K. M. Rodamo), desalegndabaro2016@gmail.com(D.D.Dangiso) \\ *Corresponding author
}

\section{To cite this article:}

Gorfu Geremew Gunsa, Kaleb Mayisso Rodamo, Desalegn Dabaro Dangiso. Determinants of Acute Diarrhoea among Children Aged 6-59 Months in Chire District, Southern Ethiopia: Unmatched Case-Control Study. Journal of Gynecology and Obstetrics.

Vol. 6, No. 2, 2018, pp. 15-25. doi: 10.11648/j.jgo.20180602.11

Received: February 23, 2018; Accepted: March 13, 2018; Published: May 9, 2018

\begin{abstract}
Background: Acute diarrhoea is a major global health problem, and the developing countries like Ethiopia bear the huge share of the burden. As of 2015, Ethiopia stands sixth among fifteen high diarrhoea death occurring countries in the world. Chire district is one of the affected areas in South Ethiopia despite the absence of scientific evidences of driving factors. Thus, this study was aimed to assess the determinants of acute diarrhoea among under-five year aged children in Chire district of Southern Ethiopia. Methods: Facility based unmatched case-control study was conducted in three purposely selected health centers. Five hundred twelve children were included into two groups, 256 cases and 256 controls. Simple random sampling technique was used to select the study subjects. Standardized structured questionnaire was used for data collection and SPSS version 20.0 for data analysis. Descriptive analysis, bivariate and multivariate logistic regression model were used to identify the associated factors. Results: The study showed that age of 6-11 months [AOR: $2.43,95 \% \mathrm{CI},(1.28,4.61)$ ], five or more persons in the house-hold [AOR: $2.69,95 \% \mathrm{CI},(1.50,4.83)$ ], inappropriate disposal of infant feces [AOR: 3.69, 95\%CI, $(1.13$, 6.49)], lack of vitamin-A supplementation [AOR: $3.44,95 \% \mathrm{CI},(1.82,6.49)$ ], maternal history of recent diarrhoea [AOR: 1.68 , $95 \% \mathrm{CI},(1.07,2.65)]$ and poor knowledge of respondents about risk factors were significantly associated with acute diarrhoea. Conclusion: Majorities of identified findings have strong association with the maternal or care-takers behavior. Thus, the responsible bodies, especially the district health office and the health extension workers are highly required to provide the intensive and regular health education on the causes and consequences of disease. In addition, vitamin-A should be supplemented as per national guideline.
\end{abstract}

Keywords: Acute Diarrhoea, Determinants, Children Aged 6-59 Months

\section{Introduction}

Acute diarrhoea is a major public health problem in the world. Next to pneumonia, it is the leading cause of death among under-five year aged children. The toll of death is high in developing countries [1, 2]. Thus, only Sub-Saharan Africa and South Asia alone accounted for $84 \%$ of diarrhoea death among under-five year aged children in 2000 and $88 \%$ in 2015 [2]. In addition, as of meta-analysis report of studies from 2012-2017 in three selected East African countries, the averaged prevalence of under-five diarrhoea was $27 \%$ [3].

Like in other developing countries, acute diarrhoea is a major health threat in Ethiopia. It stands $6^{\text {th }}$ among top 15 high diarrhoea deaths occurring countries among children under five years of age in 2015 [2]. In addition, the burden of disease distribution is significantly varied across time and places within the country. Different studies demonstrated such variations within the country. According to community based study in Jabithennan district of north Ethiopia, 21.5\% 
of under-five year aged children have experienced acute diarrhoea, similarly $14.7 \%$ in Adama district of eastern Ethiopia, 30.5\% in Arba Minch district of Southern Ethiopia, and $16.7 \%$ in Farta Wereda of North West Ethiopia [4-7].

Different factors are associated with the occurrence and distribution of acute diarrhoea throughout world. Major determinants include socio-demographic and economic factors, cultural factors, sub-optimal feeding, environmental factors, personal hygiene, contaminated complementary food feeding, treatment and feeding practice during illness, malnutrition, lack of vaccination services and micro-nutrient deficiency [1, 2, 8, 9].

Recognizing the above-mentioned global burden and the associated factors, different preventive actions have been exerted. Major preventive actions taken were community wide sanitation, safe water supply, hand washing with the soap, exclusive breast feeding and vitamin A supplementation [10-13]. Despite these efforts, still it is a big public health problem especially in developing countries [1, $2,14]$. In addition, the reduction of diarrhoea related morbidity was not appreciated in the view of millennium development goal [15].

Ethiopia has also been adopted above-mentioned intervention. In addition, the country has introduced a new program, Health Extension program, to provide comprehensive, universal, equitable and affordable health services [16]. Thus, the remarkable achievements of health service performance have been reported including significant decline of under-five death [17]. However, acute diarrhoea is still a common cause of under-five morbidity and mortality in the country $[4,5,7,18,19]$. Similarly, acute diarrhoea was the common problem of Chire district in south Ethiopia. As of 2015 annual performance report of health office, it was the top leading causes of under-five morbidity in the district [20]. However, there was no scientific evidence of causative factors. Thus, the aim of this study was to identify the potential determinants associated with the acute diarrhoea in children aged 6-59 months in this district.

\section{Methods and Materials}

\subsection{Study Area and Period}

The study was conducted in Chire district, one of the 19 districts and 4 town administrations of Sidama zone, in southern Ethiopia. It is located at about $200 \mathrm{~km}$ from Hawassa city, the capital of south region of the country and Sidama zone as well. The district includes 18 kebeles (small unit of administration), and has 148,722 total and 21,011 under-five aged populations. There were five health centres and 16 health posts in the district. This study was conducted from January to February in 2016 in three health centers of the district.

\subsection{Study Design}

Facility based unmatched case-control study design was conducted in three purposely selected health centers. In general, 512 study subjects included in two groups, 256 cases and 256 controls. Cases in this study were children aged 6-59 months who visited health centers for acute diarrhoea treatment. Children with the symptomatic diagnosis like dehydration, and with three or more loose stools without blood per day for less than two weeks was classified as cases. Controls were children aged 6-59 months who visited health centers for treatment other than acute diarrhoea.

\subsubsection{Source Population}

All children aged 6-59 months who visited health centers in Chire District during the study period.

\subsubsection{Study Population}

Selected population of children aged 6-59 months who visited- selected health centers during the study period.

\subsection{Inclusion and Exclusion Criteria}

\subsubsection{Inclusion Criteria}

Cases were the children at age 6-59 months whom were brought to health centers and diagnosed with only acute diarrhoea, and children diagnosed with other cases than acute diarrhoea were included in control of study. In addition, those whom were brought for immunization services were included as controls of study.

\subsubsection{Exclusion Criteria}

Children aged 6-59 months whose mothers or care-takers could not respond to questionnaire due to health related problems, and children with chronic diseases were not included.

\subsection{Sample Size and Sampling Techniques}

The sample size was calculated based on the assumptions of findings from different studies; hand washing practice and maternal education factors were taken from the study conducted in Arba-Minch district [21] and waste refuse method and age of index child were taken from study conducted in Eastern Ethiopia [22].

$\mathrm{P} 1=$ percent of being exposed to hand washing practice among controls.

$\mathrm{P} 2=$ percent of being exposed to hand washing practice among cases.

Sample size for poor and good hand washing practice was calculated using Epi info 7 Software.

Accordingly, sample size for each factor without nonresponse rate was 233 .

Total sample size with $10 \%$ non-respondent rate for this study was 512 .

Simple random sampling technique was used to select 512 study cases and controls. Three health centers (Abaye-Taka, Chire and Fechena) were selected purposely based on the accessibility and feasibility to study. Cases were proportionally allocated to each health center based on the annual expected number of under-five children with acute diarrhoea in its catchment. Expected number of under-five children with acute diarrhoea was $16.4 \%$ of total under-five 
aged children in the population; $16.4 \%$ is a conversion factor to obtain the estimated number of under-five children with acute diarrhoea [23]. Then, the cases expected to visit each health center within a month was calculated by dividing those expected within a year to twelve month. Finally, total cases needed from each health center were obtained as shown below (Figure 1). Then, the same numbers of controls were taken from each health centers.

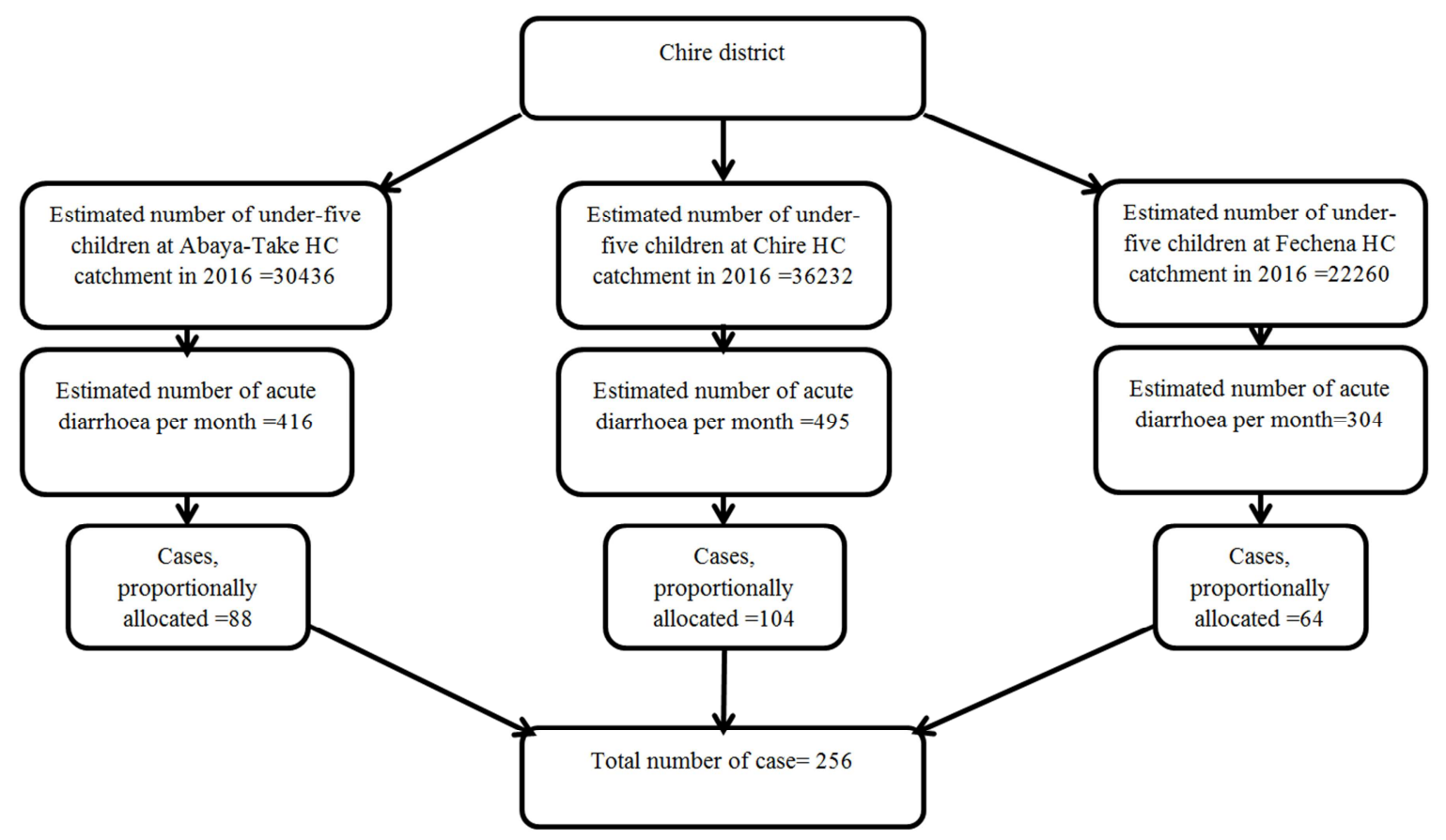

Figure 1. Schematic diagram of sampling procedure.

\subsection{Data Collection Tools:}

Structured questionnaire translated to local language, (Sidaamu afoo), was used. Ten data collectors (diploma qualified nurses) were employed for data collection and supervised by three trained health officers. Both data collectors and supervisors were trained for one and half days on data collection tool. The tool was pre-tested on $5 \%$ of sample size in similar population in adjacent health centers that were not included in the study. The finding of pretest was discussed among data collectors, supervisors and researchers in order to ensure better understanding of tools and procedures so that it was modified to final version.

\subsection{Data Management and Analysis}

SPSS version 20.0 was used for analysis. Binary logistic regression analysis was used to calculate odds Ratios (OR) with 95\% confidence intervals (CI) to estimate the association between the dependent and independent variables. To identify the relative effects of explanatory variables on the outcome variable, variables that had $p$-value less than 0.25 during bivariate analysis were carried into multivariate analysis. Finally, the findings were presented by tables, figures and narrations.

\subsection{Dependent Variable}

Occurrence of acute diarrhoea among children aged 6-59 months during the study period.

\subsection{Independent Variables}

(i) Socioeconomic characteristics: Maternal or caretakers' age, age of index child, sex of child, birth order of the child, number of under-five children, family size, occupation of the mother, occupation of the father, marital status of mother, education status of mother and father, and average monthly income of house-hold.

(ii) Environmental factors: Housing condition (floor and roof), houses shared with domestic animals, availability of a latrine, availability of hand washing facility near latrine, waste disposal system, types of water source and time from water source.

(iii) Hygiene practices: Latrine utilization, hand washing practice at critical times, method of water storage, method of water drawing, water treatment, and infant faeces disposal place, maternal history of recent diarrhoea and knowledge of respondents about risk factors.

(iv) Child health care practices: Immunization status (measles and rotavirus vaccine), breast feeding status, de-worming, vitamin-A micronutrient, duration of breastfeeding, time of initiating supplementary feeding and child feeding materials.

\subsection{Operational Definition of Terms}

(i) Acute diarrhoea: Is defined as a diarrhoea disease that causes three or more loose or watery stools in twenty 
four hours and diagnosed by health workers, and recorded on integrated community case management registration book in the health facility.

(ii) Cases: were children aged 6-59 months who visited health centers and diagnosed with acute diarrhoea by health workers, and registered on integrated community case management books with in study period.

(iii) Controls: Were children aged 6-59 months who visited the health centers for treatment other than acute diarrhoea including respiratory tract infection, otitis media, accidents and those who came for immunization service.

(iv) Determinants: Factors that have association with acute diarrhoea among children aged 6-59 months.

(v) Hand washing at critical times: Hand washing practices of mothers or care-takers of children aged 6-59 months before food preparation, before child feeding, after cleaning child's bottom and visiting latrine.

(vi) Appropriate disposal of liquid or waste water: Proper disposal of waste water in a pit prepared for it or far away from nearby houses.

(vii) Inappropriate liquid waste disposal: Improper disposal of waste water in or around house that could affect health of community including under-five children.

(viii) Index child: Was a child who was included in the study.

(ix) Good knowledge of respondents about major risk factors: Respondents who mentioned at least three major risk factors such as none vaccinated for Rota virus and measles, lack of early initiation and exclusive breast feeding, using unsafe water for drinking and food preparation, lack of hand washing practice with soap at critical times, eating un properly handled food and lack of latrine facility.

(x) Poor knowledge of respondents about major risk factors: Refers to respondents who mentioned at most two risk factors stated above, in section (ix).

\subsection{Ethical Consideration}

Ethical clearance was obtained from Institutional Review Board of Hawassa University, College of Medicine and Health Science. The permission was obtained from Chire district health office and respective health centres. Informed consent was obtained from mothers or care-takers of children after careful explanation of the purpose of study.

\section{Results}

\subsection{Socio-demographic and Economic Characteristics of Respondents and Children Aged 6-59 Months}

A total of 512 respondents, at age range of 6-59 months were included in the study providing a response rate of 100\%. Among these, 439 (85.7\%) were biological mothers and the rest were care-takers. Out of the total respondents, mothers of $147(57.4 \%)$ cases and 138 (53.9\%) controls were found in the age group of 25-34 years. The mean $( \pm$ S. D) age of both respondents of cases and controls was $25.74( \pm 4.3)$ years; $97.5 \%$ of them were married. Mothers or care-takers of $99(38.6 \%)$ cases and $97(37.8 \%)$ controls were uneducated and mothers of 28 (10.9\%) cases and 48 (18.7\%) controls had completed secondary school. Mothers or caretakers of $(79.6 \%)$ cases and $(68.4 \%)$ controls were housewives. Fathers of 67 (26.1\%) cases and $54(21.0 \%)$ controls were uneducated and father of $132(51.5 \%)$ cases and $103(40.2 \%)$ controls were farmers.

Among the index children, $295(57.6 \%)$ were male and 78 (30.54\%) cases and 99 (38.6\%) controls among index children were born first and 45 (17.5\%) cases and 58 (22.6\%) controls were born either fourth or above. The median (IQR) age of index children was 24 (25) months. In addition, out of all index children, about $52(20.3 \%)$ cases and $83(32.4 \%)$ controls were at age range of 6-12 months and 81 (31.6\%) cases and $87(34.0 \%)$ controls were at age of 36 and above months (Figure 2).

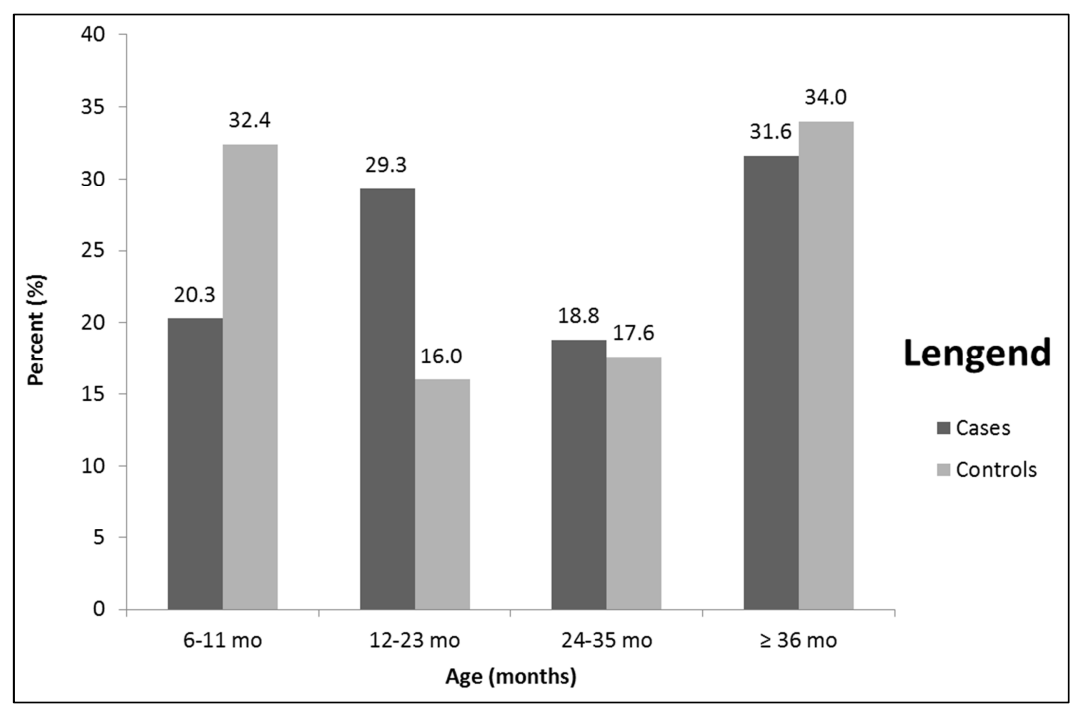

Figure 2. Age distribution of index children in cases and controls in Chire District, Southern Ethiopia, 2016. 
The mean $( \pm$ S. D) family size of all respondents was $4.86( \pm 1.49)$ persons. House-holds with family size of 5 or above constituted $75.0 \%$ among cases and $87.1 \%$ among controls whereas there were two under-five children in the house-holds of $134(52.3 \%)$ cases and $116(45.3 \%)$ controls. Mothers/care-takers of $144(56.2 \%)$ cases and $120(46.8 \%)$ controls had earned less than 500 birr per month. The median (IQR) monthly income of house-holds of both cases and controls were $500( \pm 650)$ birr.

Table 1. Socio-demographic and economic characteristics of respondents and children aged 6-59 months in Chire District, Southern Ethiopia, 2016, (n=512).

\begin{tabular}{|c|c|c|c|}
\hline Characteristic of respondents & Category & Cases Number (\%) & Controls Number (\%) \\
\hline \multirow{3}{*}{ Age (year) } & $15-24$ & $102(39.8)$ & $105(41.0)$ \\
\hline & $25-34$ & $147(57.4)$ & $136(53.1)$ \\
\hline & $\geq 35$ & $7(2.7)$ & $15(2.9)$ \\
\hline \multirow{3}{*}{ Education } & Illiterate & $99(38.7)$ & $97(37.9)$ \\
\hline & Primary school (1-8) & $129(50.4)$ & $111(43.4)$ \\
\hline & Secondary $\&$ above & $28(10.9)$ & $48(18.8)$ \\
\hline \multirow{4}{*}{ Occupation of respondent } & House wife & $204(79.7)$ & $175(68.4)$ \\
\hline & Student & $7(2.7)$ & $20(7.8)$ \\
\hline & Merchant & $31(12.1)$ & $36(14.1)$ \\
\hline & Civil servant & $14(5.5)$ & $25(9.8)$ \\
\hline \multirow{4}{*}{ Occupation of father $(\mathrm{N}=505)$} & Farmer & $132(52.2)$ & $103(40.9)$ \\
\hline & Student & $11(4.3)$ & $15(6.0)$ \\
\hline & Merchant & $81(32.0)$ & $88(34.9)$ \\
\hline & Civil servant & $29(11.5)$ & $46(18.3)$ \\
\hline \multirow{4}{*}{ Age of index child } & $6-11$ & $52(20.3)$ & $83(32.4)$ \\
\hline & $12-23$ & $75(29.3)$ & $41(16.0)$ \\
\hline & $24-35$ & $48(18.8)$ & $45(17.6)$ \\
\hline & $\geq 36$ & $81(31.6)$ & $87(34.0)$ \\
\hline \multirow{4}{*}{ Birth order of index child } & First & $78(30.5)$ & $99(38.7)$ \\
\hline & Second & $72(28.1)$ & $61(23.8)$ \\
\hline & Third & $61(23.8)$ & $38(14.8)$ \\
\hline & Fourth or above & $45(17.6)$ & $58(22.7)$ \\
\hline \multirow{2}{*}{ Number of family size in house hold } & $<5$ & $64(25.0)$ & $33(12.9)$ \\
\hline & $\geq 5$ & $192(75.0)$ & $223(87.1)$ \\
\hline \multirow{3}{*}{ Number of u5 childrenIn house hold } & 1 & $106(41.4)$ & $127(49.6)$ \\
\hline & 2 & $134(52.3)$ & $116(45.3)$ \\
\hline & $3 \&$ above & $16(6.2)$ & $13(5.1)$ \\
\hline \multirow{3}{*}{ Monthly income of house holds } & $\leq 500$ & $144(56.2)$ & $120(46.9)$ \\
\hline & $501-1000$ & $74(28.9)$ & $78(30.5)$ \\
\hline & $\geq 1001$ & $38(14.8)$ & $58(22.7)$ \\
\hline
\end{tabular}

$\mathrm{n}=$ total number of study participants.

\subsection{Environmental Health Characteristics of Respondents, Houses}

According to the participants, households of 89 (34.7\%) cases and $128(50 \%)$ controls have been using piped water sources whereas $53(20.7 \%)$ cases and $38(14.8 \%)$ controls have been using unprotected spring or well water sources. One hundred twenty five (48.8\%) of cases and 133 (52\%) controls spent less than 15 minutes (round trip) to fetch drinking water but most of the them, cases and controls, did not treat drinking water at home. In addition, house-holds of 51 (19.9\%) cases and $16(6.2 \%)$ controls did not have latrines. Among those who have latrines, $183(88.8 \%)$ cases and 197 (82.1\%) controls were without hand washing facility (Table 3 ).

Table 2. Environmental health characteristics of respondent's house (of cases and controls) in Chire District, Southern Ethiopia, 2016, ( $n=512$ ).

\begin{tabular}{|c|c|c|c|}
\hline Variables & Category & Cases Number (\%) & Controls Number (\%) \\
\hline \multirow{3}{*}{ Source of drinking water } & Piped water & $89(34.8)$ & $128(50.0)$ \\
\hline & Protected spring/well & $114(44.5)$ & $90(35.2)$ \\
\hline & Unprotected spring/well & $53(20.7)$ & $38(14.8)$ \\
\hline \multirow{2}{*}{ Treat drinking water at home } & Yes & $4(1.6)$ & $6(2.3)$ \\
\hline & No & $252(98.4)$ & $250(97.7)$ \\
\hline \multirow{2}{*}{ Availability of latrine } & Yes & $205(80.1)$ & $240(93.8)$ \\
\hline & No & $51(19.9)$ & $16(6.2)$ \\
\hline Latrine ownership $(\mathrm{n}=446)$ & Shared & $49(23.8)$ & $31(12.9)$ \\
\hline \multirow{2}{*}{ Availability of hand washing facility $(n=446)$} & Yes & $23(11.2)$ & $43(17.9)$ \\
\hline & No & $183(88.8)$ & $197(82.1)$ \\
\hline \multirow{2}{*}{ Livestock shared the same house with family } & Yes & $177(69.1)$ & $138(53.9)$ \\
\hline & No & $79(30.9)$ & $118(46.1)$ \\
\hline Housing floor material & Mud & $210(82.0)$ & $172(67.2)$ \\
\hline
\end{tabular}




\begin{tabular}{llll}
\hline Variables & Category & Cases Number (\%) & Controls Number (\%) \\
\hline & Wood & $17(6.6)$ & $41(16.0)$ \\
& Cement & $29(11.3)$ & $43(16.8)$ \\
& Thatched & $18(7.0)$ & $0(0.0)$ \\
Housing roof material & Corrugated iron sheet & $126(49.2)$ & $8(3.1)$ \\
& Wood/Grass & $112(43.8)$ & $248(96.9)$ \\
& $<15$ minute & $125(48.8)$ & $133(52.0)$ \\
Time to fetch drinkingwater (round trip) & $15-30$ minute & $113(44.1)$ & $113(44.1)$ \\
& $>30$ minute & $18(7.0)$ & $10(3.9)$ \\
Number of living room & $\leq 2$ & $181(70.7)$ & $148(57.8)$ \\
& $\geq 3$ & $75(29.3)$ & $108(42.2)$ \\
Refuse (solid waste) disposal method & Appropriate & $64(25.0)$ & $99(38.7)$ \\
& In appropriate & $192(75.0)$ & $157(61.3)$ \\
Liquid (waste water) waste disposal method & Appropriate & $189(73.8)$ & $194(75.8)$ \\
\hline
\end{tabular}

n:-refers total number of index children. Appropriate for solid waste; - disposed solid waste in pit, garbage can or Burned whereas inappropriate;- disposed of solid waste in somewhere (open filed). Appropriate for liquid waste means disposed it a manner not affecting health of people.

\subsection{Hygiene and Child health Care Practices Among Families of Children Aged 6-59 Months}

In a total of 445 respondents who have latrine, only mothers or care-takers of $37(18 \%)$ cases and $99(41.2 \%)$ controls have washed their hands after using latrine, out of which $21(56.8 \%)$ and $49(49.5) \%$ of mothers or care-takers of cases and controls used only water to wash their hands, respectively (Table 4). Of 248 index children whose age was less than 24 months, about $64(51.6 \%)$ cases and $22(17.7 \%)$ controls had started supplementary feeding before six months of age (Figure 3 ).

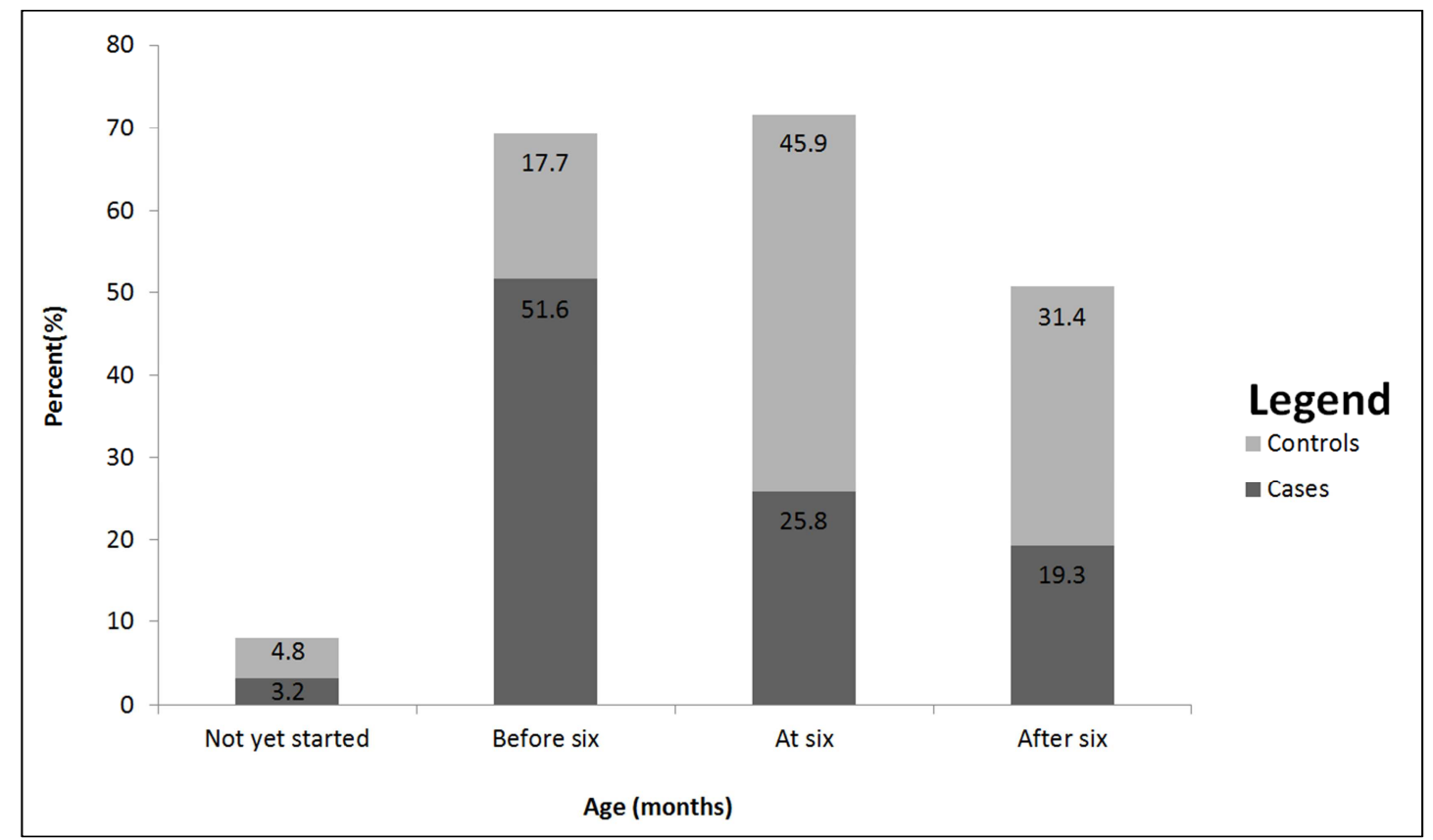

Figure 3. Age of children (cases and controls) at which supplementary feeding was started.

Table 3. Hygiene and child health care practices of respondents in Chire District, Southern Ethiopia, 2016.

\begin{tabular}{llll}
\hline Variables & Category & Cases Number (\%) & Controls Number (\%) \\
\hline \multirow{2}{*}{ Used covered water storage } & Yes & $240(93.7)$ & $253(98.8)$ \\
& No & $16(6.2)$ & $3(1.1)$ \\
Means of drawing drinking water from storage container & Pouring & $249(97.2)$ & $251(98.0)$ \\
& Dipping & $7(2.7)$ & $5(1.9)$ \\
Dispose infant faeces in the latrine & Yes & $114(44.5)$ & $185(32.0)$ \\
Mother/care-taker always washed hands after visiting latrine (n & No & $142(55.5)$ & $71(27.1)$ \\
$=445)$ & No & $37(18.0)$ & $99(41.2)$ \\
Materials used to wash hand after latrine use (n=136) & Water only & $168(82.0)$ & $141(58.8)$ \\
& Water and soap/ash & $21(56.8)$ & $49(49.5)$ \\
Mother/care-taker washed hands before preparing food & Yes & $245(43.2)$ & $50(50.5)$ \\
Prepared child food separately using cleaned and separate utensils & No & $11(2.1)$ & $247(48.2)$ \\
\hline
\end{tabular}




\begin{tabular}{llll}
\hline Variables & Category & Cases Number (\%) & Controls Number (\%) \\
\hline$(\mathrm{n}=502)$ & No & $164(65.1)$ & $141(56.4)$ \\
Child can feed by himself $(\mathrm{n}=502)$ & Yes & $95(37.7)$ & $98(39.2)$ \\
& No & $157(62.3)$ & $152(60.8)$ \\
Washing of child's hand before feeding $(\mathrm{n}=1$ 193) & Always & $66(69.5)$ & $75(76.5)$ \\
& Sometimes & $29(30.5)$ & $23(23.5)$ \\
Material used to wash child's hand $(\mathrm{n}=193)$ & Water only & $90(94.7)$ & $78(79.6)$ \\
& Water and soap/ash & $5(5.3)$ & $20(20.4)$ \\
& Hand & $86(27.8)$ & $66(26.4)$ \\
Child feeding practices $(\mathrm{n}=309)$ & bottle & $35(22.3)$ & $31(20.0)$ \\
& cup & $81(26.2)$ & $72(23.3)$ \\
Time the supplementary feeding started $(\mathrm{n}=248)$ & spoon & $88(28.4)$ & $107(34.6)$ \\
& Not yet started & $4(3.2)$ & $6(4.8)$ \\
& Before six month & $64(51.6)$ & $22(17.7)$ \\
\hline
\end{tabular}

$\mathrm{n}$ : refers to total number of index children for a variable about which data has been collected.

Among the 451 index children in the age group of $9-59$ months, $62.7 \%$ cases and $28.2 \%$ controls were not vaccinated for measles and $140(54.7 \%)$ cases and 190 (74.2\%) controls did not receive vitamin-A. Mothers or care-takers of 120 (46.8\%) cases and 184 (71.9\%) controls have poor knowledge about major risk factors of acute diarrhoea (Table 5).

Table 4. Hygiene and child health care practices of respondents in Chire District, Southern Ethiopia, 2016, (n=512).

\begin{tabular}{|c|c|c|c|}
\hline Child care variables & Category & Cases Number $(\%)$ & Controls Number (\%) \\
\hline \multirow{2}{*}{ Maternal history of recent acute diarrhoea } & Yes & $151(59.0)$ & $82(32.0)$ \\
\hline & No & $105(41.0)$ & $174(68.0)$ \\
\hline \multirow{3}{*}{ Current breast feeding status of child $(n=248)$} & Exclusive breast feed & $4(3.2)$ & $7(5.6)$ \\
\hline & Partially breastfeed & $116(93.5)$ & $109(87.9)$ \\
\hline & Not breast feed & $4(3.2)$ & $8(6.5)$ \\
\hline \multirow[b]{2}{*}{ Child received measles vaccine $(n=451)$} & Yes & $77(33.3)$ & $146(66.4)$ \\
\hline & No & $145(62.7)$ & $62(28.2)$ \\
\hline \multirow{3}{*}{ Child received vit-A (in the last six month) } & Yes & $104(40.6)$ & $53(20.7)$ \\
\hline & No & $140(54.7)$ & $190(74.2)$ \\
\hline & Unknown & $12(4.7)$ & $13(5.1)$ \\
\hline \multirow{3}{*}{ Child receive Rota-virus vaccine } & Yes & $146(28.5)$ & $176(34.4)$ \\
\hline & No & $102(19.9)$ & $75(14.6)$ \\
\hline & Unknown & $8(1.6)$ & $5(1.0)$ \\
\hline \multirow{2}{*}{ Child received De-worming (24-59 months) $(n=262)$} & Yes & 44 (16.7) & $73(27.8)$ \\
\hline & Unknown & $17(6.4)$ & $13(4.9)$ \\
\hline \multirow{2}{*}{ Knowledge of respondents about major risk factors of acute diarrhoea } & Good & $136(53.2)$ & $72(28.1)$ \\
\hline & Poor & $120(46.8)$ & $184(71.9)$ \\
\hline
\end{tabular}

\subsection{Determinants of Acute Diarrhoea Among Children Aged 6-59 Months}

The associations of different factors such as sociodemographic, environmental, hygiene and child health care practices with acute diarrhoea among children aged 6-59 months were assessed using bivariate and multivariate logistic regression analysis. All variables with p-value $<0.25$ in bivariate logistic regression analysis were selected for multivariate logistic regression model in order to assess relative effect of explanatory variables on acute diarrhoea and to control the possible effect of cofounders. According to bivariate logistic regression analysis, most of the variables have a significant association with occurrence of acute diarrhoea (Table 5). Among socio-demographic and economic factors education and occupation of respondent, education of father, age of index child, birth order of index child, family size and average monthly family income had significant association $(\mathrm{p}<0.05)$ with acute diarrhoea in bivariate analysis. However, in multivariate regression analysis, only age of index child [AOR: $2.43,95 \%$ CI: (1.28, 4.61] and number of family size [AOR: $2.69,95 \% \mathrm{CI}:(1.50$, 4.83)] showed strong association.

Among the environmental factors latrine availability, having livestock and sharing the same houses $(p<0.001)$, source of drinking water, hand washing facility near the latrine, floor type of living houses, number of living rooms and solid waste disposal method $(\mathrm{p}<0.05)$ had a significant association. But due to some socioeconomic and behavioural factors in the study setting, there was no variable with the significant association by multivariate regression analysis.

Among hygiene practices disposal method of infant or child faeces, hand washing after using latrine, maternal history of recent acute diarrhoea, respondents' knowledge about major risk factors of acute diarrhoea $(p<0.001)$ and preparing child food separately using cleaned separate 
materials had significant association with occurrence of diarrhoea by bivariate logistic analysis.

Among these, infants' or Childs' faeces disposal method [AOR: $3.69,95 \% \mathrm{CI}(1.13,5.93)]$ and maternal history of recent diarrhoea [AOR: $1.68,95 \% \mathrm{CI}:(1.07,02.65)]$ were significantly associated with occurrence of child-hood diarrhoea by multivariate logistic analysis.

Vitamin-A supplementation [AOR:3.44, 95\% CI (1.82, 6.49)] and knowledge about major risk factors [AOR:4.0, $95 \%$ CI: $(2.52,6.35)]$ were only factors among those child health care practices which showed significant association with occurrence of acute diarrhoea by multivariate regression analysis.

This study showed that child age had strong association with acute diarrhoea. Accordingly, the odds of getting acute diarrhoea among children in age group of 6-11 months were 2.4 times more compared to those found in age group of 36 and above months [AOR: 2.43, 95\% CI: $(1.28,4.61]$. In children who lived in house-hold with family size of five or above persons, the odds of getting diarrhoea was 2.6 times more as compared with those children living in family size of less than five persons [AOR: 2.69, 95\%CI:(1.50, 4.83)].

Disposal of infant or child faeces also showed strong association with occurrence of acute diarrhoea. Accordingly, children whose faeces disposed in somewhere (other than latrines) had 3.6 times more odds of getting diarrhoea as compared to those children whose faeces were disposed in the latrines [AOR:3.69, 95\% CI $(1.13,5.93)]$.

Children who were not supplemented with vitamin-A within last six month had 3.4 times more odds of getting acute diarrhoea compared to those children who received vitamin-A [OR: 3.44, 95\% CI:(1.82, 6.49)].

Children whose mothers or care-takers had recent history of diarrhoea had 1.6 times more odds of getting diarrhoea as compared with their counter parts [AOR: 1.68, 95\% CI: $(1.07,02.65)]$. The odds of getting acute diarrhoea among children belonging to mothers or care-takers who had poor knowledge about major risk factors of acute diarrhoea was four times more compared to those children living with mothers or care-takers of having good knowledge [AOR:4.0, $95 \%$ CI: $(2.52,6.35)]$.

Table 5. Factors associated with occurrence of acute diarrhoea among children aged 6-59 months who visited selected Health Centers in Chire District, Southern Ethiopia, $2016(n=512)$.

\begin{tabular}{|c|c|c|c|c|c|}
\hline Variables & Category & $\begin{array}{l}\text { Cases Number } \\
(\%)\end{array}$ & $\begin{array}{l}\text { Controls } \\
\text { Number (\%) } \\
\end{array}$ & $\operatorname{COR}(95 \% \mathrm{CI})$ & AOR $(95 \%$ CI $)$ \\
\hline \multirow{4}{*}{ Age of children } & $6-11$ & $52(20.3)$ & $83(32.0)$ & $1.48(0.93,2.35)$ & $2.43(1.28,4.61)$ \\
\hline & $12-23$ & 75 (29) 3 & $41(16.0)$ & $0.50(0.31,0.82)$ & $0.54(0.28,1.03)$ \\
\hline & $24-35$ & $48(18.8)$ & $45(17.6)$ & $0.87(0.52,1.45)$ & $0.98(0.5,1.89)$ \\
\hline & $\geq 36$ & 81 (31.6). & $87(34.0)$ & 1 & 1 \\
\hline \multirow{2}{*}{ Number of persons in the house-hold } & $<5$ & $64(25.0)$ & $33(12.9)$ & 1 & 1 \\
\hline & $\geq 5$ & $192(75.0)$ & $223(87.1)$ & $2.25(01.41,3.57)$ & $2.69(1.50,4.83)$ \\
\hline \multirow{2}{*}{ Dispose infant faeces in the latrine } & Yes & $114(44.5)$ & $71(27.1)$ & 1 & 1 \\
\hline & No & $142(55.5)$ & $185(72.3)$ & $2.09(1.44,3.02)$ & $3.69(1.13,5.93)$ \\
\hline \multirow{3}{*}{ Child received vit-A } & Yes & $104(40.6)$ & $53(20.7)$ & 1 & 1 \\
\hline & No & $140(54.7)$ & $190(74.2)$ & $2.63(1.82,3.95)$ & $3.44(1.82,6.49)$ \\
\hline & Unknown & $12(4.7)$ & $13(5.1)$ & $2.12(0.907,4.98)$ & $3.19(1.13,8.99)$ \\
\hline \multirow{2}{*}{ Maternal history of recent acute diarrhoea } & Yes & $151(59.0)$ & $82(32.0)$ & $1.47(1.02,2.11)$ & $1.68(1.07,2.65)$ \\
\hline & No & $105(41.0)$ & $174(68.0)$ & 1 & 1 \\
\hline \multirow{2}{*}{$\begin{array}{l}\text { Knowledge of respondents about major risk } \\
\text { factors of acute diarrhoea }\end{array}$} & Good & $130(50.8)$ & $72(28.1)$ & 1 & 1 \\
\hline & Poor & $126(49.2)$ & $184(71.9)$ & $2.64(1.83,3.80)$ & $4.00(2.52,6.35)$ \\
\hline
\end{tabular}

$\mathrm{n}$;-refers to total number of index children. 1; - indicates reference category for each variable.

\section{Discussion}

The associations of socio-demographic, environmental, hygiene and child health care practices with acute diarrhoea were assessed using multivariate logistic regression model. The study showed that age of index child, number of persons in the house-hold, infant faeces disposal place, vitamin-A supplementation, maternal history of recent diarrhoea and knowledge of respondents about major risk factors were significantly associated with occurrence of acute diarrhoea among children.

It was well documented that most diarrhoea episodes occur during first two years of life of children [24-26]. In this study, children within age category of 6-11 months have 2.4 times more odds of getting acute diarrhoea as compared with children in the age category of 36 or above months. This finding is consistent with a number of different studies in developing countries. According to the study finding in India, the risk of diarrhoea morbidity was higher at age ranges of 611 months than others [27] Similarly, it is also in line with the different studies in Ethiopia [18, 22, 28-30]. On the other hand, it contrasts with the results of studies conducted in Kushitia in Bangladesh, Uganda, Burundi and BenishangulGumuz region of North West Ethiopia where children at age category of 12-24 months were more at risk than others [18, 31-33]. In this study the reason might be, at this age child's inborn immunity has become lost, supplementary feeding practices increases, crawling or walking to be started so that child could pick up dirty or contaminated food and could ingest.

The finding revealed that family size has a significant association with acute diarrhoea. Where, children living in house with 5 and more persons had 2.6 folds of odds to get diarrhoea compared to those with less than five persons. 
Similarly, studies elsewhere reported a significant association of diarrhoea and number of children in household [34-36]. This, most probably, is due to as the number of children increases, the probability of contaminating the kitchen utensils including the water and food containers increases. In addition, as the number of children increases in household the maternal care could decrease, and thus children might easily exposed to risk factors of diarrhoea disease.

In the rural community, disposal of infant faeces in the compound or in open field has a significance health impact on the children as well as on others. It is a suitable chain to spread acute watery diarrhoea easily within short period of time once it happened. According to this study, disposal of infant faeces had a significant association with occurrence of diarrhoea. Improper disposal of infant faeces had 3.6 times more odds of getting diarrhoea among children aged 6-59 months than those disposed the faeces in the latrine in proper way. This is consistent with the study finding in Derashe district of South Ethiopia [37]. This is also comparable with other studies on prevalence and associated factors of diarrhoea in Benishangul-Gumuz region of North West Ethiopia and Dejen districts north west Ethiopia [18, 38]. This could be attributed to the fact that in the study area, the community had farms occupied with constant crops so that they dispose infant's faeces in the compound or open field as well as in the farms in a way that is accessible to the children while playing in such contaminated fields.

Vitamin-A is an essential micronutrient for children aged 6-59 months to prevent different under-five diseases including diarrhoea [9, 39]. It is essential micronutrient to decrease the severity of diarrhoea by improving the gut integrity [40, 41]. In addition, if not taken once in every six month interval period, the child becomes susceptible for occurrence of acute diarrhoea [42]. According to this study, children who did not receive vitamin-A in the last six months had 3.4 times more odds of getting diarrhoea as compared with those who received it. This is comparable with the findings of studies conducted in Enemay and Dejen districts of north west Ethiopia [38, 43]. But it contrasts with 2011 Ethiopia demography and health survey report, according to which vitamin-A supplementation was not associated with acute diarrhoea [24].

Maternal history of recent diarrhoea was identified as a risk factor for acute diarrhoea. According to this study, children from mothers with history of recent diarrhoea had 1.6 times more odds of getting diarrhoea compared to those from mothers that did not report recent history of diarrhoea during the study. This is comparable with study finding in Mecha and Shebedino district, and Debre brehan town in Ethiopia [19, 42, 44]. These studies showed that children who were living with mothers who had recent history of diarrhoea had high odds of getting diarrhoea than those children whose mothers did not report recent history of diarrhoea.

Lack of knowledge on risk factors of diarrhoea showed the significant association with occurrence of acute diarrhoea. As a result, children from mothers or care-takers who had poor knowledge about major risk factors of acute diarrhoea had four times more odds of getting diarrhoea as compared to those children who were born from mothers or care-takers of good knowledge about risk factors. Similarly, different studies have reported that the maternal or caretakers' knowledge has a significant preventive and control effect on diarrhoea disease [35, 45-47].

\section{Conclusion}

Age of index children, family size, infants' faeces disposal places, vitamin-A deficiency, maternal or caretakers history of recent diarrhoea and maternal or caretakers knowledge about diarrhoea were the major factors for acute diarrhoea among children aged 6-59 months in the district. Thus, majorities of identified findings have strong association with the maternal or care-takers behavior except vitamin-A supplementation. Thus, the responsible bodies especially the district health office maternal and child health work process and the health extension workers are highly required to take the contextual behavioral factors of acute diarrhoea and should provide the intensive and regular health education on the causes and consequences of disease. In addition, vitamin-A should be supplemented as per national guideline.

\section{Acknowledgements}

We would like to acknowledge Hawassa University, College of Medicine and Health Science, School of Public and Environmental Health for financial support. In addition, we would like to thank Chire district health office for their kind support during data collection.

\section{Authors' Contribution}

Gunsa GG, has conceived the research idea and designed the study. Rodamo KM and Dangiso DD have contributed in designing the study, analysis and writing the paper.

\section{Conflict of Interest}

The authors have no conflict of interest.

\section{References}

[1] UNICEF. The State of the World'S Children 2016. A fair chance for every child. 2016.

[2] UNICEF. One is too many. Ending child deaths from pneumonia and diarrhea One is too many Ending child deaths from pneumonia and diarrhea. New York, NY 10017, USA: 2016.

[3] O. E. ELMI, R. (II) P. DIOSO. Prevalence of Diarrheal Diseases Among Children under Five Years in East African Countries from 2012-2017 ASEAN Journal on Science and Technology for Development,. 2017; 3 (1). 
[4] Anteneh ZA, Andargie K, Tarekegn M. Prevalence and determinants of acute diarrhea among children younger than five years old in Jabithennan District, Northwest Ethiopia,. BMC Public Health 2017; 17 (99).

[5] RegassaW, Lemma S. Assessment of Diarrheal Disease Prevalence and Associated Risk Factors in Children of 6-59 Months Old at Adama District Rural Kebeles, Eastern Ethiopia, January/2015. 2016:581-8.

[6] Mohammed S, Tamiru D. The Burden of Diarrheal Diseases among Children under Five Years of Age in Arba Minch District, Southern Ethiopia, and Associated Risk Factors: A Cross-Sectional Study. Hindawi Publishing Corporation International Scholarly Research Notices. 2014.

[7] Gedamu G, Kumie A, Haftu D. Magnitude and Associated Factors of Diarrhea among Under Five Children in Farta Wereda, North West Ethiopia. Quality in Primary Care 2017; 25 (4):199-207.

[8] Robert Black. Solutions to defeat a global Killer. Path, Johns Hopkins University, Epidemiology of Diarrhea 1 Diseases, 2007.

[9] WHO. Guideline: vitamin A supplementation in infants and children 6-59 months of age. r. Geneva,: 2011.

[10] Carl E. Taylor, William B. Greenough I. Control of diarrheal diseases. Annu Rev Public Health. 1989; 10:221-44.

[11] Enzley S, Barros F. A Global Review of Diarrhea 1 Disease Control. New York, N. Y., USA: UNICEF, 1997.

[12] PATH \&Tearfund. DIARRHEA DIALOGUES From Policies to Progress A call for urgent action to prevent the biggest killer of children in sub-Saharan Africa. 2012.

[13] EPPI-Centre Social Science Research Unit Institute of Education University of London. What factors affect sustained adoption of clean water and sanitation technologies? A Systematic Review of Literature Protocol. 2013.

[14] Ferrer S, Strina A. A hierarchical model for studying risk factors for child-hood diarrhea: A case-control study in a middle-income country, International Journal of Epidemiology 2008; 37:805-15.

[15] Kumar SG SL. Diarrhea 1 Diseases in Developing Countries: A Situational Analysis. Kathmandu Univ Med J. 2012; 38 (2):83-8.

[16] FMoH. Introduction of new health extension programs 2002/2003.

[17] CSA, MoH. Ethiopia Mini Demographic and Health Survey 2014. Addis Ababa, Ethiopia: 2014.

[18] Mihrete TS, Alemie GA, Teferra AS. Determinants of childhood diarrhea among under-five children in BenishangulGumuz Regional State, North West Ethiopia. BMC Pediatrics. 2014; 14 (102).

[19] Mamo A, Hailu A. Assessment of prevalence and related factors of diarrhea disease among under-five children in Debrebrehan Referal Hospital, Debrebrehan Towan, Amhara Region, Ethiopia Open Access Library Journal. 2013.

[20] Chire District Health Office. Annual Performance Report of Health Service. Chire, South Ethiopia: 2015.

[21] Mohammed S, Tilahun M, Tamiru D. Morbidity and associated factors of diarrhea 1 diseases among under-five children in Arba-Minch district, Southern Ethiopia,. Science journal of public health 2012; 1 (2):102-6.

[22] Mengistie B, Berhane Y, Worku A. Prevalence of diarrhea and associated risk factors among children under-five years of age in Eastern Ethiopia: A cross-sectional study Open journal of preventive medicine. 2013; 3 (7):446-53.

[23] FMoH. Woreda based national plan Addis Ababa, Ethiopia: 2016.

[24] Central Statistical Agency [Ethiopia] and ICF International. Ethiopia Demographic and Health Survey 2011. Addis Ababa, Ethiopia and Calverton, Maryland, USA: 2012.

[25] Central Statistical Agency (CSA) [Ethiopia] and ICF. Ethiopia Demographic and Health Survey 2016. Addis Ababa, Ethiopia, and Rockville, Maryland, USA: CSA and ICF.: 2016.

[26] Gupta A, Sarker G, Rout AJ, Mondal T, Pal R. Risk Correlates of Diarrhea in Children Under 5 Years of Age in Slums of Bankura, West Bengal. J Glob Infect Dis. 2015; 7 (1):23-9.

[27] Ahmed SF, Farheen A, Muzaffar A, Mattoo G. Prevalence of diarrhea 1 disease, Its seasonal and age variation in underfives in Kashmir, India.. India journal of health science (Qassim). 2008; 2 (2):126-33.

[28] Gebru T, Taha M, W. K. Prevalence of diarrhea in under-five children among health extension model And non-model house-holds in Sheko district rural community, Southwest Ethiopia.. Science journal of public health. 2013; 1 (5):230-4.

[29] Mamo A, Hailu A. Assessment of Prevalence and Related Factors of Diarrheal Diseases among Under-Five Year's Children in Debrebirehan Referral Hospital, Debrebirehan Town, North Shoa Zone, Amhara Region, Ethiopia. Open Access Library Journal. 2013.

[30] Mohammed S, Tilahun M, Tamiru D. Morbidity and associated factors of diarrheal diseases among under five children in Arba-Minch district, Southern Ethiopia, 2012 Science Journal of Public Health. 2013; 1 (2):102-6.

[31] Khatun A, Rahman SS, Rahman H, Hossain S. A cross sectional study on prevalence of diarrhea 1 disease and nutritional status among children under 5 -years of age in Kushtia, Bangladesh. Science Journal of Public Health. 2013; $1(2): 56-61$.

[32] Senyonga R, Twebaze F, Muwonge R, R. M. Determinants of acute diarrhea in children aged $0-5$ years in Uganda. East African Medical Journal x. 2009; 86. (11).

[33] Diouf K, Tabatabai P, Rudolph J, Marx M. Diarrhea prevalence in children under five years of age in rural Burundi: an assessment of social and behavioural factors at the household level. Global Health Action,. 2014; 7 (1):24895.

[34] Bado AR, Susuman AS, Nebie EI. Trends and risk factors for childhood diarrhea in sub-Saharan countries (19902013): assessing the neighborhood inequalities. Global Health Action. 2016.

[35] AI Mohammed, Zungu L. Environmental health factors associated with diarrhea 1 diseases among under-five children in the Sebeta town of Ethiopia. Southern African Journal of Infectious Diseases 2016; 31 (4):122-9.

[36] Tambe AB, Nzefa LD, Nicoline NA. Childhood Diarrhea Determinants in Sub-Saharan Africa: A Cross Sectional Study of Tiko-Cameroon. Challenges,. 2015,; 6: 229-43. 
[37] Godana W, Mengiste B. Environmental factors associated with acute diarrhea among children under five years of age in derashe district, Southern Ethiopia. Science Journal of Public Health. 2013; 1 (3):119-24.

[38] Belete T, Getu D, A. W. Child-hood diarrheal disease among under- five children at Dejen District, North west Ethiopia. American journal of health research. American journal of health research. 2014; 2 (4):177-81.

[39] Mayo-Wilson E, Imdad A, Herzer K, Yawar Yakoob MY, Bhutta ZA. Vitamin A supplements for preventing mortality, illness, and blindness in children aged under 5: systematic review and meta-analysis. BMJ 2011; 343.

[40] Villamor E, Fawzi WW. Effects of Vitamin A supplementation on immune responses and correlation with clinical outcomes,. Clinical Microbiology Reviews,. 2005; 3:446-64.

[41] Marpaung M, Supriatmo, Atan Baas A. Effect of vitamin A on severity of acute diarrhea in children. Paediatr Indones,. 2013; 53 (3):125-31.

[42] Dessalegn M, Kumie A, Tefera W. Predictors of under-five child-hood diarrhea: Mecha district, West Gojam, Ethiopia.. Ethiop journal health development. 2011; 25 (3):174-232.
[43] Ayele A, Awoke W, Tarekegn M. Assessment of diarrheal disease prevalence and associated factors among children under-five in Enemay district, Northwest Ethiopia.. Global journal of Medical Research. 2014; 17 (3).

[44] Tamiso A, Yitayal M, Awoke A. Prevalence and determinants of child-hood diarrhea among graduated house-holds, in rural area of Shebedino district, Southern Ethiopia. Science journal of public health. 2013; 2 (3):243-51.

[45] Kijakazi O, Mashato H, Malebo M, Emil M, Emanuel P. Prevalence, one week incidence and knowledge on causes of diarrhea: House-hold survey of under-five and adults in Mkuranga district, Tanzania. BMC public health. 2014 (19):985.

[46] Kumar SG SL. Diarrhea 1 Diseases in Developing Countries: A Situational Analysis. Kathmandu Univ Med J. 2012; 38 (2):83-8.

[47] Tarekegn M, F. E. A case-control Study on determinants of diarrhea 1 morbidity among under-five children in Wolaita Soddo Town, Southern Ethiopia.. Ethiopia journal of health development 2012; 26 (2). 